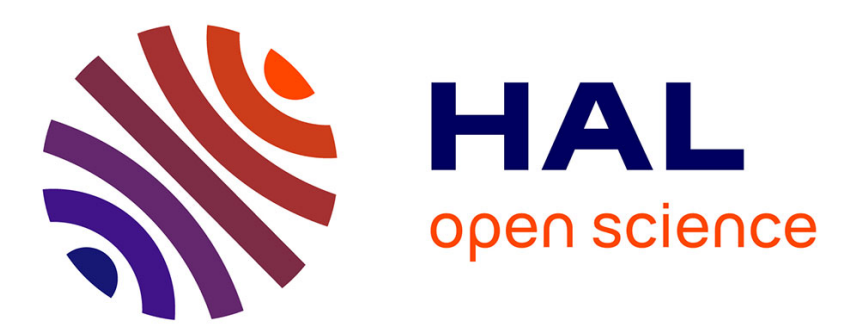

\title{
A partial differential equation model predictive control strategy: Application to autoclave composite processing
}

Pascal Dufour, Youssoufi Touré, Dennis J Michaud, Prasad S. Dhurjati

\section{To cite this version:}

Pascal Dufour, Youssoufi Touré, Dennis J Michaud, Prasad S. Dhurjati. A partial differential equation model predictive control strategy: Application to autoclave composite processing. Computers \& Chemical Engineering, 2004, 28 (4), pp.545-556. 10.1016/j.compchemeng.2003.08.007 . hal00351748v2

\author{
HAL Id: hal-00351748 \\ https://hal.science/hal-00351748v2
}

Submitted on 21 Jan 2009

HAL is a multi-disciplinary open access archive for the deposit and dissemination of scientific research documents, whether they are published or not. The documents may come from teaching and research institutions in France or abroad, or from public or private research centers.
L'archive ouverte pluridisciplinaire HAL, est destinée au dépôt et à la diffusion de documents scientifiques de niveau recherche, publiés ou non, émanant des établissements d'enseignement et de recherche français ou étrangers, des laboratoires publics ou privés. 
This document must be cited according to its final version which is published in a journal as:

P. Dufour ${ }^{1}$, Y. Touré ${ }^{2}$, D.J. Michaud ${ }^{3}$, P.S. Dhurjati ${ }^{3}$, "A partial differential equation model predictive control strategy: Application to autoclave composite processing", Computers and Chemical Engineering, ISSN: 0098-1354 28(4), pp. 545-556, 2004. http://dx.doi.org/10.1016/j.compchemeng.2003.08.007

All open archive documents of Pascal Dufour are available at: http://hal.archives-ouvertes.fr/DUFOUR-PASCAL-C-3926-2008

The professional web page (Fr/En) of Pascal Dufour is: http://www.lagep.univ-lyon1.fr/signatures/dufour.pascal

1

Université de Lyon, Lyon, F-69003, France; Université Lyon 1;

CNRS UMR 5007 LAGEP (Laboratoire d'Automatique et de GEnie des Procédés),

43 bd du 11 novembre, 69100 Villeurbanne, France

Tel +33 (0) 472431845 - Fax +33(0) 472431699

http://www-lagep.univ-lyon1.fr/ http://www.univ-lyon1.fr http://www.cnrs.fr

2

Université d'Orléans,

UPRES EA 2078 LVR (Laboratoire de Vision et de Robotique ),

63 Av de Lattre de Tassigny, 18020 Bourges Cedex, France

http://www.bourges.univ-orleans.fr/rech/lvr/

3

University of Delaware,

Department of Chemical Engineering,

Newark, DE 19716, USA

http://www.udel.edu 


\title{
A Partial Differential Equation Model Predictive Control Strategy: Application to Autoclave Composite Processing
}

\author{
Dufour P. ${ }^{\mathrm{a}, 1}$, Michaud D.J. ${ }^{\mathrm{b}}$, Touré Y. ${ }^{\mathrm{c}}$, Dhurjati P.S. ${ }^{\mathrm{b}}$ \\ ${ }^{\mathrm{a}}$ LAGEP, Université Claude Bernard Lyon 1, UMR CNRS 5007 \\ 43, Bd. du 11 Novembre 1918, 69622 Villeurbanne Cedex, France \\ ${ }^{\mathrm{b}}$ Department of Chemical Engineering, University of Delaware \\ Newark, DE 19716, USA \\ ${ }^{\mathrm{c}}$ LVR, Université d'Orléans, UPRES EA 2078 \\ 63, Av. de Lattre de Tassigny, 18020 Bourges Cedex, France
}

\begin{abstract}
A general framework for a partial differential equation (PDE) model predictive control problem is formulated. A first principle model of the system, described by a semi-linear PDE system with boundary control, is employed in a model predictive control (MPC) framework. Here, the aim is to determine, off-line (i.e. without pro-
\end{abstract}


cess measurement), the theoretical optimal behavior of the process that will be used during on-line MPC. Input and output constraints are handled in the optimization task using a non-linear programming method. This strategy is evaluated for the optimization of processing temperatures during the manufacture of thick-sectioned polymer composite laminates. The off-line optimization task consists of determining the optimal temperature profile, otherwise known as the cure cycle. Moreover, for this particular process, the existence of a feasible constrained optimization problem is discussed through the design of a constraint bound.

Key words:

Model predictive control, distributed parameter model, nonlinear programming, trajectory optimization, composite manufacturing, autoclave curing process.

\section{Introduction}

The framework presented here deals with a model predictive control (MPC) problem based on a semi-linear parabolic PDEs system characterized by boundary control. Transport reaction phenomena with significant diffusive and convective phenomena are typically characterized by severe nonlinearities and spatial variations and are naturally described by partial differential equations. Examples of such processes include tubular reactors, packed bed reactors, absorption columns, drying or polymerization processes. The large number of real applications described by such PDE models constitutes the motivation for this work. Moreover, as modeling becomes more and more accurate, the use of complex nonlinear PDE models is increasing. Unfortunately, in con-

$\overline{1}$ Corresponding author: Email dufour@lagep.univ-lyon1.fr; Phone (33) 4724318 78, Fax (33) 472431699 
trol theory, due to the complexity of the problem, relatively few studies are devoted to the control of processes explicitly characterized by a PDE model, especially in the nonlinear case. Various methods are proposed for control of such distributed parameter systems but there is no general framework yet. In order to implement a low order model based controller with a computer, the original PDE model is usually simplified into an ordinary differential equation (ODE) model. Such a finite dimension approximation is based on finite differences, finite volume method, orthogonal collocation or Galerkin's method. Recently other studies have utilized properties of the initial PDE system before finite dimension controller synthesis. Christofides developed order reduction by partitioning the eigenspectrum of the operator of the PDE system $[9,15]$ and methods based on approximate inertial manifold for spatial discretization of the PDE $[8,1]$. Other approaches for controller synthesis of nonlinear PDE systems are based on symmetry groups, infinitesimal generators and invariant conditions $[31,17]$. In $[19,43]$, a finite dimensional controller is obtained through model reduction based on various methods: singular value decomposition, Karhunen-Loéve expansion or eigenfunction method. In [2], stability conditions for closed loop control of a linear PDE with a finite dimension controller are given in the time and frequency domains through semigroup analysis. In [42], based on semigroup theory, proofs were given for the closed loop stability of PI control for a linear PDE system.

In regards to MPC, it has become an advanced control strategy widely used in industry $[37,36]$. In any MPC formulation, the model is essential: it aims to predict future process behaviors in an optimization procedure. Historically, models chosen in practical applications were time domain, input/output representations or obtained through step or impulse responses. Even if these kinds of models are easily obtained, their drawback is a lack of confidence in modeling 
complex system behaviors like those observed in the autoclave curing process, where nonlinearities of the reaction and distributed aspect are important. Few papers where MPC was applied for PDE system exist $[13,32,41,20]$. In these works, accurate high order dimension models are accounted for. The drawback with such accurate PDE models is that their online resolution during the optimization task may prohibit the implementation of the real-time control strategy. To reduce the dimension of the nonlinear elliptic model, KarhunenLoéve expansion combined with weighted residuals method was used to get the finite dimensional model used in the MPC framework [3].

Our previous studies in parabolic PDE model predictive control dealt with the online control of processes. In [11], the aim was to follow the specification of the maximum amount of polluted gas released at the catalytic reverse flow reactor outlet combined with the minimization of energy consumption. In [12], a trajectory tracking for a drying process had to be ensured. In these studies, we first focussed on real-time control issues since the PDE model involved in the online optimization task may have a large size [11] and may then prohibit implementation of any model predictive control strategy. To tackle this issue, we developed the control strategy in two steps (the strategy is detailed in $[11,12])$. Step one (off-line) is to choose a behavior based on the nonlinear PDE model. At this point, no process measurement is needed to find the entire behavior since this is an off-line procedure. Step two (online), based on a linearized PDE model computed online around the behavior determined off-line and based on process measurements, is to adjust the process behavior to the optimal behavior determined online. One of the advantages of this strategy is to replace the online (accurate) resolution of the nonlinear PDE model by the online (approximate) resolution of a smaller size linearized PDE model. This allows to reduce the calculation time dedicated to the resolution of the model 
used in the online MPC procedure. Application of this two-step control strategy helps to show the robustness of this approach with respect to modeling errors $[11,12]$ and input disturbances as well [11]. Since we first emphasized our work on real-time control aspects (step two of our procedure), the off-line behavior was not optimized.

The objective of this paper is to focus on step one of the control strategy and to provide an approach for the determination of the off-line theoretical optimal process behavior that will be used during our on-line control strategy $[11,12]$. The first part of the paper will describe the general aspects of thick-sectioned composites manufacturing techniques and the autoclave molding process. The manufacture of thick-sectioned polymer composite laminates was chosen as a model system for its non-linear response to control actions, the complex interactions between processing time and final part quality, and the availability of a relatively accurate process model. In the second half of the paper, the proposed PDE model predictive control approach is developed in a general framework. Constraints handling and the nonlinear programming method are detailed. Finally, the approach is evaluated for the optimization in the autoclave molding process. Simulation runs are discussed with an emphasis on the importance and design of the constraint bound.

\section{Background on Thick-Sectioned Composites}

Despite many challenges in producing thick composites of adequate quality, the need for composite parts with thick cross-sections and high strength continues to increase [29]. The composite components of bridge structures, tank and submarine hulls, and airplanes can require cross-sections greater than 1 
inch. The improper manufacture of these thick composite laminates can lead to large thermal gradients (and subsequent delaminations), long processing times, and inadequate mechanical properties. Manufacturing thick-sectioned thermoset composites can be difficult due to the significant amount of heat generated by the reacting resin and the low thermal conductivity of the composite. Since the center of the composite is furthest from the mold surfaces, the center is severely heat transfer limited and the heat generated by the exothermic polymerization reaction raises the internal temperature of the composite. If these high temperatures within the composite part are not successfully controlled, they can lead to degradation of the polymer and possibly large internal voids from the vaporization of resin components. In addition, past research into stresses generated within thick composite laminates [6] established the importance of "inside-out" curing in order to reduce residual stresses within the laminate and improve product strength. These stresses are generated when the outside surfaces of the composite polymerize significantly before the center. Therefore, the extent of cure of the resin at the surfaces of the composite should be kept as low as possible before the center is cured. However, enough heat needs to be added to the system to initiate resin cure at the center and to later ensure that the polymerization reaction proceeds to completion throughout the composite.

\subsection{Vacuum Assisted Autoclave Layup Molding}

A number of different processing techniques exist for manufacturing thermoset composites; however, only a few are successfully used in industry for the production of thick-sectioned laminates and other polymer composites [29]. His- 
torically, thick-sectioned composites have been manufactured by the vacuum assisted autoclave molding process. Consequently, nearly all research in the optimization and control of thick-sectioned composites have involved this process technique (e.g. $[34,38,39,10,23,28,24,22,7,21,40])$. While the use of other processing techniques, such as resin transfer molding (RTM), is increasing for the manufacture of thick-sectioned composites, the optimization of processing temperatures is still a concern and methods for identifying optimal temperature trajectories, such as the approach presented here, are very relevant [30]. Vacuum assisted autoclave molding used in this work can produce strong, quality composites with higher fiber volume percentage and low void fractions, but unfortunately it requires a high capital cost autoclave unit with accurate temperature and pressure control. Another limitation is that processing times can be on the order of four to six hours.

Autoclave layup processing begins by laying up a number of materials on the tool surface, as shown in Figure 1 (a more extensive process description can be found in [33]). The bottom tool surface shapes the bottom surface of the composite. The final composite shape is further defined by a metal "picture frame" along its edges and a top caul plate. Layers of "prepreg" are placed in the mold and become the composite portion of the layup. Prepregs are sheets of unidirectional fibers or woven fabrics impregnated with partially cured resin. Thermocouples can be placed within the mold for feedback information, but not without a detrimental effect on the part's mechanical strength properties. Vacuum is achieved by covering mold and breather cloth structure with a sealed, impermeable vacuum bag. Once the mold assembly is sealed within the autoclave and vacuum has begun, the autoclave temperature and pressure control programs are initiated. The temperature of the air circulating in the autoclave is increased to reduce the viscosity of the resin and eventually initi- 
ate the polymerization of the resin. The pressure within the autoclave is raised to aid the evacuation of excess resin from prepreg to consolidate the laminate. Consolidation is an important aspect of autoclave molding as it determines the part's final thickness, fiber content, and void fraction. While some of the optimization and control strategies developed for the autoclave molding process have focused primarily on achieving good part consolidation, minimizing thermal and stress gradients within thick composites is an important issue and can be adequately decoupled from the consolidation problem.

\subsection{Cure Cycle Optimization}

The processing conditions used to produce a thermoset composite are often collectively called a "cure cycle". A cure cycle is often presented as the profile (or trajectory) of temperatures used to initiate cure (polymerization) within the composite laminate and bring it to completion. For autoclave molding, the cure cycle may also include an autoclave pressure profile used to aid consolidation of the composite laminate. In this work, only the internal temperature and extent of cure profiles of the composite are modeled, but the approach can be extended in the future with the addition of a resin viscosity model to identify the optimal autoclave pressure profile for consolidation.

Prior to introduction of computers, the cure cycle for thick-sectioned composites was developed from the recommended cycle through experimental trial and error methods [38]. While still sometimes used, a trial and error procedure can be expensive, time-consuming, and non-optimal even under the supervision of manufacturing expert. Computers have provided a much more systematic and hopefully improved means of modifying cure cycles to suc- 
cessfully produce thick-sectioned composite laminates. Early simulations were primarily used to manually generate different cure cycles for thick-sectioned composites to determine better processing conditions than those suggested by the resin's manufacturer for thinner laminates $[25,18,6,10,23]$. While this trial and error approach often yielded better quality composites, identifying the optimal cure cycle was unlikely. Furthermore, due to the complexity of the heterogeneous composite system, the accuracy of the models used is an important concern.

To evaluate cure cycles more systematically, a number of researchers have simulation-based optimization strategies that are implemented off-line. Loos and Nagendra [26] used a dynamic programming optimization technique to reduce thermal lag and temperature overshoots. Martinez [28] used a hillclimbing algorithm to identify a temperature trajectory that resulted in an $80 \%$ decrease in heat up time for a $2.54 \mathrm{~cm}$ thick composite by using aggressive heating initially followed by a significant cooling phase before the final heating stage. In Pillai et al. [33,34], a look-ahead strategy was employed to evaluate the effect of different control actions during processing on the pre-defined penalty function. This method decreased by $33 \%$ the processing time recommended by the prepreg manufacturer while also improving product quality. Michaud et al. [30] used a similar methodology but with a global optimization algorithm designed to account for model parameter variabilities during the off-line optimization of a cure cycle.

On-line control of thick-sectioned composite processes has primarily been accomplished through the use of expert systems to adjust autoclave temperature and pressure settings according to pre-defined heuristic rules (e.g. $[39,24,10,22])$. Choi and Lee [7] combined an expert system approach with traditional on-line control by dividing the composite process into three stages, 
each with its own distinct control strategy to account for process variability. Using a trained artificial neural network (ANN) as a model, Joseph et al. [21] developed an on-line model-based optimization and control strategy for the autoclave molding process. This work was later extended to use a shrinkinghorizon model predictive control strategy [40]. In this paper, we use the off-line MPC framework presented here for the determination of the theoretical optimal cure cycle.

\section{Modeling and Problem Statement}

\subsection{Modeling}

A model of the curing process for thermosetting composites was first presented by Loos and Springer [25]. Since then, various aspects of modeling the autoclave curing process have been investigated by numerous authors (see [4] for a review). The core of the model used here was first developed by Bogetti [5]. The governing equations are the general anisotropic heat conduction equation coupled with the kinetics relating the rate of reaction $\alpha$ (also named fractional degree of cure) to the temperature $T$. Since the 2-D problem has demonstrated that temperature gradients were negligible in lateral direction, only 1-D phenomena across the thickness are accounted for. The critical dimension is therefore across the thickness $z$ and, by assuming the symmetry of the process, is divided in two parts $\mathcal{D}_{c}, \mathcal{D}_{t}$ from the center of the composite to the interface between the autoclave and the mold assembly (Figure 2). $\mathcal{D}_{c}$ represents the composite portion of the system and $\mathcal{D}_{t}$ represents the tooling portion. Major change in the model used here (Pillai et al. [33]) is 
the boundary condition (convective in [5]). Pillai et al. lumped, at the edge of the composite, the heat transfer resistance in the autoclave, the bag, the fabric and the mold in a single layer, referred to as the tooling. The accuracy of the model was improved considerably by explicitly accounting for the heat capacity of the tooling. Concerning the kinetic model of the polymerization reaction, it is modeled using an empirical Arrhenius-type rate law. The curing process can therefore be described by the following nonlinear parabolic PDE system $\left(\mathcal{S}_{N L}\right)[33]$ with boundary control $T_{a}$.

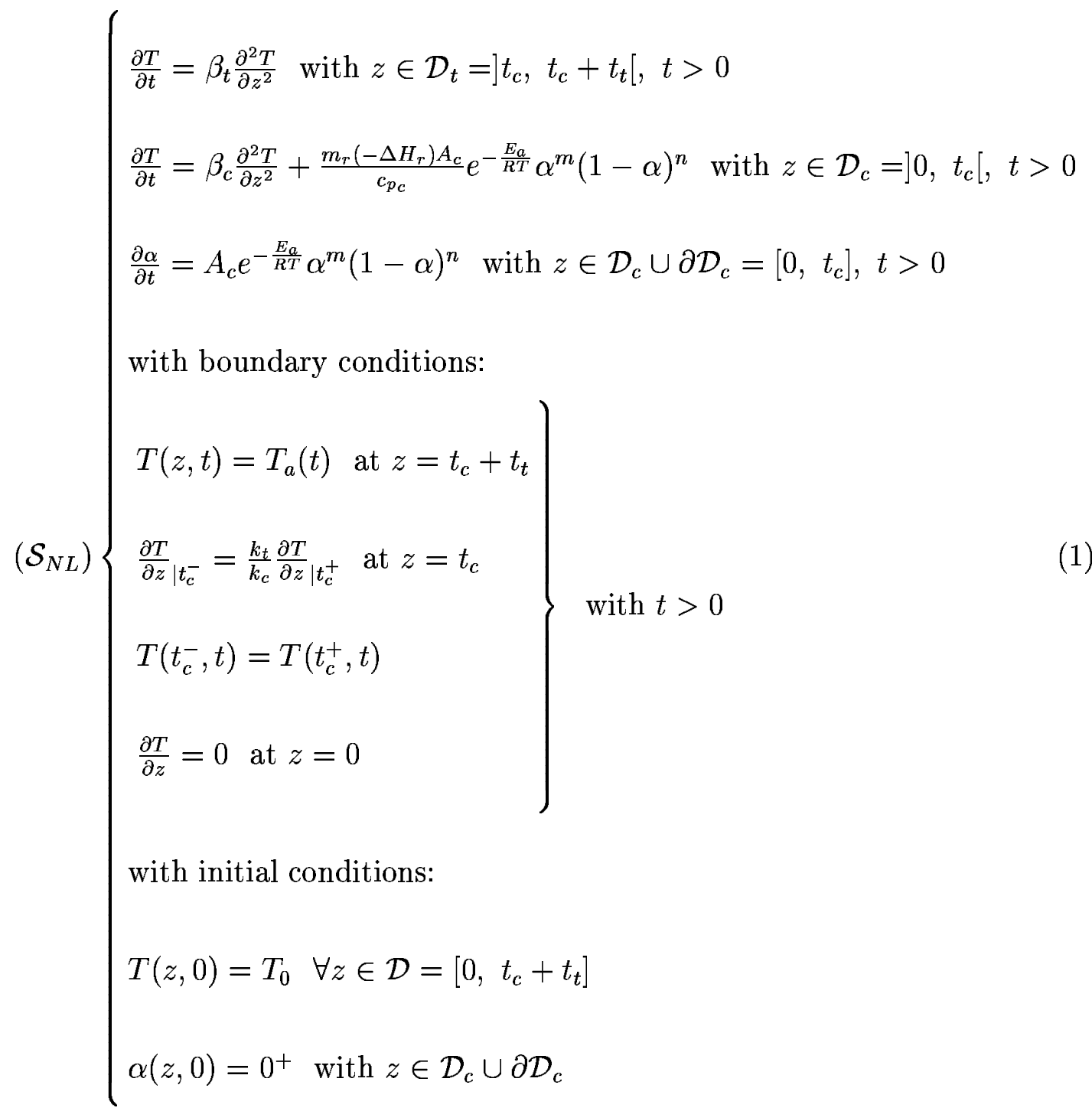

with the parameter values given in appendix. 


\subsection{Constrained Optimization Problem Formulation}

The optimization problem is to find, at each sample time $k\left(t=k T_{e}, T_{e}\right.$ is the sampling time), the value of the manipulated variable $T_{a}$ such that the cure cycle is completed in minimum time. The performance index is chosen such that it maximizes the fractional degree of cure rate at the center of the composite:

$$
\min _{T_{a}(k)} J\left(T_{a}(k)\right)=\frac{1}{\dot{\alpha}^{2}(0, k)}
$$

To ensure that the resulting cure cycle can realistically be implemented, constraints are introduced to define the range of temperatures and to limit the heating $\left(\Delta T_{a \max }\right)$ and cooling $\left(\Delta T_{a \min }\right)$ rates of the autoclave:

$$
\left\{\begin{array}{l}
T_{a \min } \leq T_{a}(k) \leq T_{a \max } \\
\Delta T_{a \min } \leq \frac{T_{a}(k)-T_{a}(k-1)}{T_{e}} \leq \Delta T_{a \max }
\end{array}\right.
$$

Due to the lack of stress model available previously underlined, addressing the inside-out curing issue is handled through constraints for the temperature difference between the surface and center of the composite:

$$
\Delta T_{\min } \leq T\left(t_{c}, k\right)-T(0, k) \leq \Delta T_{\max }
$$

This constrained optimization procedure is iterated until the polymer is entirely cured at $k=k^{*}$ :

$$
\alpha\left(z, k^{*}\right) \geq \alpha_{f}, \quad \forall z \in \mathcal{D}_{c}
$$




\section{Off-line Optimization Methodology}

\subsection{Distributed Parameter Model Considered}

One considers the following class of first principle model described by a semilinear one dimensional parabolic PDE system with boundary control $\left(\mathcal{S}_{N L}\right)$ :

$$
\left(\mathcal{S}_{N L}\right)\left\{\begin{array}{l}
\frac{\partial \underline{x}(z, t)}{\partial t}=A_{d} \underline{x}(z, t)+F_{d}(\underline{x}(z, t)) \quad \forall z \in \Omega, t>0 \\
A_{b} \underline{x}(z, t)+B_{b} u(t)=0 \quad \forall z \in \partial \Omega, t>0 \\
\underline{y}(t)=C \underline{x}(z, t) \quad \forall z \in \partial \Omega \cup \partial \Omega, t>0 \\
\underline{x}(z, 0)=\underline{x}_{0} \quad \forall z \in \Omega \cup \partial \Omega
\end{array}\right.
$$

where $z$ is the independent space variable, $\Omega$ is the spatial domain and $\partial \Omega$ its boundary, $t$ is the independent time variable. $x$ is the state vector in a Hilbert space $X=L_{2}(\Omega), u$ is the control or manipulated variable in $\mathbb{R}$ and $\underline{y}$ is the vector of $p$ outputs or controlled variables in $\mathbb{R}^{p} . A_{d}, A_{b}$ and $C$ are bounded linear operators, $F_{d}$ is a vector of nonlinear functions and $B_{b}$ is a matrix of suitable dimensions.

\subsection{Model Predictive Formulation}

One of the numerous advantage of MPC formulation as a constrained optimization problem is that a large number of control problems can be formulated. It covers trajectory tracking for a controlled variable, minimization of any economic function, minimization of energy supply under technical spec- 
ifications, etc [14]. Therefore, one can consider the following general task of minimizing, under some constraints, the nonlinear cost function $J$ (also named performance index):

$$
\min _{\tilde{u}} J(\tilde{u})=\sum_{j} f(\underline{y}(j), u(j-1)) \quad \forall j \in \mathcal{J}_{1}^{N_{p}}=\left\{k+1, \ldots, k+N_{p}\right\}
$$

where $k$ is the actual discrete time index, $j$ is the discrete time index, $N_{p}$ is the receding horizon and $\mathcal{J}_{1}^{N_{p}}$ is the future discrete time window. $\tilde{u}$ is the sought sequence of the future manipulated variable of the process $u$ that is classically tuned as follows:

$$
\tilde{u}=[\ldots u(j) \ldots]^{T} \quad \forall j \in \mathcal{J}_{0}^{N_{c}-1}=\left\{k, \ldots, k+N_{c}-1\right\}
$$

and:

$$
u(j)=u\left(k+N_{c}-1\right) \forall j \in \mathcal{J}_{N_{c}}^{N_{p}-1}=\left\{k+N_{c}, \ldots, k+N_{p}-1\right\}
$$

The model $\left(\mathcal{S}_{N L}\right)$, which links $u$ to $\underline{y}$, is therefore directly used in the performance index involved in (7). The optimization procedure has also to account for:

- Constraints on the magnitude and velocity of the manipulated variable:

$$
\left\{\begin{array}{l}
u_{\min } \leq u(j) \leq u_{\max } \quad \forall j \in \mathcal{J}_{0}^{N_{p}-1}=\left\{k, \ldots, k+N_{p}-1\right\} \\
\Delta u_{\min } \leq u(j)-u(j-1) \leq \Delta u_{\max } \quad \forall j \in \mathcal{J}_{0}^{N_{p}-1}
\end{array}\right.
$$

to ensure that the manipulated variable has a physical meaning.

- $n$ general output constraints:

$$
c_{i}(\underline{y}(j), u(j-1)) \leq 0 \quad \forall j \in \mathcal{J}_{1}^{N_{p}}, \forall i \in \mathcal{I}_{1}^{n}=\{1, \ldots, n\}
$$

due to operating specification, product quality, security, etc. 
This formulation leads to the solution of a constrained nonlinear optimization problem based on a nonlinear dynamic distributed parameter model with boundary control. This off-line optimization is repeated at the next sample time $k$ and stopped at the final time $k=k^{*}$ when a particular event is reached:

$$
0 \leq g\left(\underline{y}\left(k^{*}\right), u\left(k^{*}\right)\right)
$$

\subsection{Constraints Handling}

Nonlinear programming is the general case where the objective function and constraint function may be nonlinear, and is the most difficult of the optimization problems [16]. Indeed, there is no general agreement on the best approach and much research is still to be done. Historically, the earliest developments were sequential minimization methods based on the use of penalty and barrier functions. Even if they are not entirely efficient, they are simple to apply. Other popular Lagrange-Newton methods, like sequential quadratic programming (SQP), are local approaches and are used in MPC formulations. Methods proposed here to handle input and output constraints in the off-line constrained optimization problem are now detailed.

\subsubsection{Transformation Method for Input Constraints Handling}

The problem is to find a method to handle magnitude and velocity constraints on the manipulated variable. Since it is the tuning parameter, an easy method is the use of the following transformation method based on a sigmoid function $h$ depicted in Figure 3. 


$$
\left\{\begin{array}{l}
u(j)=h(p(j))=h_{\mathrm{moy}}+h_{\mathrm{amp}} \tanh \left[\frac{p(j)-h_{\mathrm{moy}}}{h_{\mathrm{amp}}}\right] \\
p(j) \in \mathbb{R}
\end{array}\right.
$$

with:

$$
\left\{\begin{array}{l}
h_{\min }=\max \left[u_{\min }, u(j-1)+\Delta u_{\min }\right] \\
h_{\max }=\min \left[u_{\max }, u(j-1)+\Delta u_{\max }\right] \\
h_{\mathrm{moy}}=\frac{h_{\max }+h_{\min }}{2} \\
h_{\mathrm{amp}}=\frac{h_{\max }-h_{\min }}{2}
\end{array}\right.
$$

Seeking the sequence $\tilde{p}$ of the unconstrained parameter $p$ always ensures the magnitude and velocity rate constraints ${ }^{2}$ check for the manipulated variable.

\subsubsection{Exterior Penalty Method for Output Constraints Handling}

In order to account for output constraints, one adopts the exterior penalty method used in nonlinear programming where a positive defined weighted penalty term is added to the initial cost function $J$ :

$$
\left\{\begin{array}{l}
J_{t o t}(\tilde{u})=J(\tilde{u})+J_{e x t}(\tilde{u}) \\
J_{e x t}(\tilde{u})=\sum_{j}\left(\sum_{i} w_{i} \max ^{2}\left(0, c_{i}(\underline{y}(j), u(j-1))\right)\right) \quad \forall j \in \mathcal{J}_{1}^{N_{p}}, \forall i \in \mathcal{I}_{1}^{n}
\end{array}\right.
$$

where $w_{i}$ is an adaptive positive defined weight: it augments when the related constraint tends to be satisfied. The penalty method transforms the problem

$\overline{2}$ Acceleration rate can also be accounted for with this transformation. 
into an unconstrained problem by substituting a penalty function for the constraints. Solution of the resulting sequence of unconstrained problem tends to a constrained minimum [27]. Roughly speaking, for any constraint $c_{i}$ not satisfied (i.e. when $c_{i}(.,)>$.0 ), a positive term penalizes the minimization task. This forces the optimizer to minimize $J_{e x t}$ and therefore forces the violated constraint to be satisfied. Moreover, one of the advantages of the method is that it can handle unfeasible problem without disturbing the overall resolution [11]. Therefore, the solution proposed during the iterative optimization task may be anywhere in the parameters space.

\subsection{Final Penalized Optimization Problem}

Finally, combining the transformation method for the constraints on the manipulated variable and the exterior penalty method for the constraints on the controlled variable, the final penalized optimization problem to be solved off-line is the following one:

$$
\left\{\begin{array}{l}
\min _{\tilde{p}} J_{t o t}(\tilde{p})=\sum_{j \in \mathcal{J}_{1}^{N_{p}}}\left(f(\underline{y}(j), p(j-1))+\sum_{i \in \mathcal{I}_{1}^{n}} w_{i} \max ^{2}\left(0, c_{i}(\underline{y}(j), p(j-1))\right)\right) \\
\tilde{p}=\left[\ldots f^{-1}(u(j)) \ldots\right]^{T} \forall j \in \mathcal{J}_{0}^{N_{c}-1} \\
p(j)=p\left(k+N_{c}-1\right) \forall j \in \mathcal{J}_{N_{c}}^{N_{p}-1} \\
\text { and subject to model }\left(\mathcal{S}_{N L}\right) \text { resolution. }
\end{array}\right.
$$

This nonlinear problem can now be solved by any unconstrained optimization algorithm. 


\subsection{Off-line Optimization Algorithm}

Widely known for its robustness and convergence properties, the LevenbergMarquardt's algorithm is used to minimize the cost function, where the sequence $\tilde{p}$ is determined at each sample instant $k$ by the iteration procedure:

$$
\tilde{p}^{n+1}=\tilde{p}^{n}-\left(\nabla^{2} J_{t o t}^{n}+\lambda I\right)^{-1} \nabla J_{t o t}^{n}
$$

where $\nabla J_{t o t}^{n}$ and $\nabla^{2} J_{t o t}^{n}$ are respectively the performance index gradient and the performance index hessian with respect to $\tilde{p}^{n}$ at the iteration $n$. $\nabla J_{\text {tot }}^{n}$ is explicitly provided into the control algorithm and the classical approximation for $\nabla^{2} J_{t o t}^{n}$ as well [16].

\section{Off-line Optimization of Cure Cycle}

In this section, the final optimization problem is stated and results of optimization of the cure cycle are discussed with a particular emphasis on the design of a constraint bound.

\subsection{Final Constrained Optimization Problem Formulation}

From the constrained optimization problem formulated in section 3.2 , one can define the problem involved in the proposed optimization procedure. The model-based performance index is: 


$$
\left\{\begin{array}{l}
\min _{\tilde{T}_{a}} J\left(\tilde{T}_{a}\right)=\sum_{j} \frac{1}{\left[\dot{\alpha}^{2}(0, j)\right]} \quad \forall j \in \mathcal{J}_{1}^{N_{p}} \\
\tilde{T}_{a}=\left[T_{a}(k) \ldots T_{a}\left(k+N_{c}-1\right)\right]^{T}
\end{array}\right.
$$

The constraints dealing with implementable value for manipulated variable are:

$$
\left\{\begin{array}{l}
T_{a \min } \leq T_{a}(j) \leq T_{a \max } \quad \forall j \in \mathcal{J}_{0}^{N_{p}-1} \\
\Delta T_{a \min } \leq \frac{T_{a}(j)-T_{a}(j-1)}{T_{e}} \leq \Delta T_{a \max } \quad \forall j \in \mathcal{J}_{0}^{N_{p}-1}
\end{array}\right.
$$

The constraints dealing with controlled variables:

$$
\Delta T_{\min } \leq T\left(t_{c}, j\right)-T(0, j) \leq \Delta T_{\max } \quad \forall j \in \mathcal{J}_{1}^{N_{p}}
$$

The procedure is iterated until $k=k^{\star}$ when the condition given in Eq. (5) is satisfied and the composite is judged to be fully cured.

\subsection{Results}

In order to reach the desired optimization objectives, two different series of runs have been carried out under the following conditions:

- The first series involves the "trial-feedback" ${ }^{3}$ design of the bound $\Delta T_{\max }$ to get a correct fractional degree of cure crossover.

- The second set of runs shows the influence of the prediction horizon over the cure cycle.

3 "Trial-feedback" terminology is preferred to the usual "trial-error" terminology since results in one simulation are used into the next one to improve results, therefore acting as a "feedback loop". 


\subsubsection{Simulation Conditions}

Simulations have been run under the following conditions:

- The sample time $T_{\boldsymbol{e}}$ is $1 \mathrm{~min}$.

- The distributed parameter model is discretized by a finite difference method with 4 nodes into $\mathcal{D}_{t}$ and 3 nodes into $\mathcal{D}_{c}$. Simulations with more nodes led to the same MPC results.

- Constraints bounds for the manipulated variable are:

$$
\begin{aligned}
T_{a \max } & =130^{\circ} \mathrm{C} \\
T_{a \min } & =10^{\circ} \mathrm{C} \\
\Delta T_{a \max } & =+4{ }^{\circ} \mathrm{C} . \mathrm{min}^{-1} \\
\Delta T_{a \min } & =-4^{\circ} \mathrm{C} . \mathrm{min}^{-1}
\end{aligned}
$$

- Initial given constraints bounds for the difference of temperature between the center and the top of the polymer are:

$$
\begin{aligned}
& \Delta T_{\max }=+10^{\circ} \mathrm{C} \\
& \Delta T_{\min }=-10^{\circ} \mathrm{C}
\end{aligned}
$$

- Initial temperature is $28^{\circ} \mathrm{C}$.

- Procedure stops for $\alpha_{f}=0.99$.

- Moreover, it is assumed that a composite with acceptable material properties will be produced if the fractional degree of cure crossover between the surface and center of the composite laminate happens before $\alpha_{\max }=0.5$ [34]. This value has a strong impact over results and leads to design the shape of the difference of temperature constraint bound.

- The software is implemented on a UltraSPARC $200 \mathrm{MHz}$ workstation. 


\subsubsection{Influence of the Temperature Difference Upper Constraint Bound}

To initially see how the bound of the temperature difference upper constraint affects the final cure cycle performance (i.e. the fractional degree of cure crossover), a first run is performed with $\left(N_{p}, N_{c}\right)=(25,1)$. The resulting cure cycle is shown in Figure 4. As it can be seen in Figure 5, the constraints put on the temperature difference between the surface and center of the composite are satisfied during the first 80 minutes. One can see that the solution is optimal until 80 minutes, since the saturation of the upper constraint limits the augmentation of $T_{a}$ naturally fixed by the performance index (the higher the autoclave temperature, the faster the cure). Then, the temperature at the surface reaches a sufficient level and the exothermic reaction can start: heat is released and the composite's surface temperature increases at a higher rate than at the center of the polymer, where the reaction has not yet begun. Consequently, since the temperature difference upper constraint was previously saturated, it can not be fulfilled anymore between 82 and 96 minutes. From 92 minutes, the surface reaction becomes more and more completed (Figure $6)$, hence less heat is created and the difference of temperature between surface and center decreases. Then, temperature at the center reaches the minimum required to start the reaction. Now, heat is trapped at the center of the composite where temperature becomes higher than at the center: the lower constraint is now no longer satisfied (Figure 5). Finally, the fractional degree of cure crossover happens at 0.92 instead of below 0.5 as desired (Figure 6).

By tuning the prediction horizon, one could expect to improve constraints checking and decreasing the fractional degree of cure crossover below its given limit. However, this is not the case because of the constrained optimization setup. Since the reaction has to be as fast as possible, the autoclave temper- 
ature has to be emphasized as much as possible. Therefore, given the initial temperature difference upper constraint bound and the diffusion phenomena, the temperature at the surface of the composite will always (i.e. for any $N_{p}$ ) reach the minimum extent necessary to start the reaction before the center of the composite will. Most importantly, the surface of the composite is cured significantly when the reaction at the center of the composite begins. Therefore, one has to enforce the difference of temperature between surface and center to decrease when surface reaction starts. Finally, through the "trial-feedback" strategy, the following bound for the difference of temperature upper constraint is designed has a funnel shape constraint [35] depicted in Figure 7. It allows the temperature profile inside the polymer to be more homogeneous when the reaction starts, hence improving the inside-out cure. This bound is adopted for the following runs.

\subsubsection{Influence of the Prediction Horizon}

The prediction horizon tuning parameter, $N_{p}$, is adjusted to determine its influence on the optimized cure cycle. In this case, the control horizon, $N_{c}$, is set to 1 to reduce the computational burden. Simulations show that $N_{c}$ tuning has less impact over the final cure cycle than $N_{p}$ tuning does. Among all the runs performed, 3 of them leading to different behaviors are depicted here: $N_{p}=15, N_{p}=25, N_{p}=45$. The obtained cure cycle is depicted in Figure 8. One can clearly see the influence of the designed upper constraint bound on the temperature difference between the surface and center of the composite laminate. Compared to the previous cure cycle (Figure 4), the autoclave temperature is no longer continually increasing until the maximum autoclave temperature bound is reached. In order to satisfy the temperature difference 
constraint, the autoclave temperature needs to level off or even cool somewhat to ensure that the surface's fractional degree of cure does not greatly surpass that of the center. The advantage of cooling stages within an autoclave molding cure cycle has been reported by a number of other researchers $[26,23,28,22]$. The real impact of the prediction horizon can be seen when $N_{p}$ is tuned to a relatively small value $\left(N_{p}=15\right)$. For such a small value, both difference of temperature constraints cannot be completely satisfied as seen in Figure 9. The funnel shape upper constraint is accounted for too late and subsequently a high autoclave temperature is reached (Figure 8). The surface of the composite heats up well before the center does and the fractional degree of cure crossover appears too late (0.75) as shown in Figure 10. Although this cure cycle would not produce a quality composite, this cure cycle is the fastest of the three runs (133 minutes) due to the higher autoclave temperatures.

By increasing the prediction horizon to a relatively large value $\left(N_{p}=45\right)$, constraints are always satisfied in Figure 9. While the resulting cure cycle appears to be a valid solution, less heat is provided to the composite than in the two other runs (Figure 8). Because of the lower processing temperatures, the reaction at the center of the composite occurs at a later time. Consequently, the fractional degree of cure crossover is delayed in time (cure cycle is the slowest of the three cases) and occurs too late at 0.75 (Figure 10). A composite manufactured with this cure cycle would most likely not have the desired mechanical properties. These two optimization runs clearly indicate that a compromise has to be found between a fast cure cycle and one with a good fractional degree of cure crossover.

By tuning the prediction horizon to an intermediate value $\left(N_{p}=25\right)$, a successful cure cycle is found with a processing time of 156 minutes (Figure 8). The constraints are always satisfied (Figure 9) and the fractional degree of 
cure crossover occurs before 0.5 (Figure 10). This cure cycle is comparable to the 170 minute cure cycle found for the same composite system by Pillai et al. using local criteria optimization [34].

\subsubsection{Table of results}

We present the following table to summarize the influence of the tuning of the prediction horizon over various aspects of the application (control horizon is first always tuned to 5):

- the value of degree of cure when the degree of cure crossover happens (it is requires to occure before $\alpha_{\max }=0.5$ ),

- the simulated processing time (that is required to be as short as possible),

- the CPU time needed to find the entire cure cycle.

\begin{tabular}{l||c|c|c|c|c}
\hline Prediction horizon $N_{p}(-)$ & 15 & 20 & 25 & 30 & 35 \\
\hline \begin{tabular}{l} 
Control horizon $N_{c}(-)$ \\
\hline \hline
\end{tabular} & 5 & 5 & 5 & 5 & 5 \\
\hline $\begin{array}{l}\text { Value of the degree of cure when the } \\
\text { degree of cure crossover happens (-) }\end{array}$ & 0.72 & 0.53 & 0.495 & 0.67 & 0.73 \\
\hline $\begin{array}{l}\text { Simulated processing time (min) } \\
\text { CPU time for the entire cycle (min.s) }\end{array}$ & 1.46 & 1.47 & 1.34 & 1.40 & 1.34 \\
\hline
\end{tabular}

One can see that prediction horizon tuning mostly influences the cure cycle results than the calculation time: indeed, one can see the balance reached by the optimal tuning of $N_{p}=25$ since the degree of cure crossover happens at 0.495 whereas the processing time is the longest of these runs (162 min). In the meantime, the calculation time is always around 1 min $40 \mathrm{~s}$. Concerning 
the influence of the control horizon, as expected, it strongly influences the calculation time: 1362 min were needed with $\left(N_{p}, N_{c}=25,25\right)$.

\section{Conclusion}

A general MPC strategy is proposed for the off-line determination of the theoretical optimal process's behavior used in our two-step MPC strategy $[11,12]$. The particularity of this process is that it is based on a nonlinear parabolic PDE model. The first principle model of the system involved in the optimization task is described by a semi-linear parabolic distributed parameter system with boundary control. A detailed MPC procedure is given. Constraints on the manipulated variable are handled through a hyperbolic transformation to ensure that these constraints are continually satisfied. Constraints on the controlled variable are accounted for through an exterior penalty method. It facilitates the constraints handling and the optimization resolution as well, since solution provided during the iterative optimization procedure may be anywhere in the parameters space.

This framework was used to optimize the processing temperature profile necessary to produce a quality thick-sectioned composite laminate in a minimum amount of time. The cure cycle optimization was complicated by the lack of an accurate model of internal residual stresses to predict the final quality of the composite. Instead, constraints were added to the predictive framework to control the temperature difference between the center and surface of the composite. A constant maximum bound for the temperature difference, however, was not sufficient to generate a good quality composite laminate (i.e. with a low fractional degree of cure crossover). Alternatively, a funnel shape func- 
tion was used for the upper constraint to ensure that the temperature profile within the composite is more homogeneous when the resin at the center of the composite begins to react. By tuning the prediction horizon of the system, an optimal cure cycle was found to manufacture a quality composite in 162 minutes.

\section{$7 \quad$ Appendix}

Parameters of autoclave cure simulation are:

\begin{tabular}{l|c|c|c}
\hline Property & Name & Value & Unit \\
\hline \hline Thermal conductivity & $k_{c}$ & 0.23793 & $W . \mathrm{m}^{-1} \cdot \mathrm{K}^{-1}$ \\
Polymer density & $\rho_{c}$ & 1890 & $\mathrm{~kg} \cdot \mathrm{m}^{-3}$ \\
Polymer thermal conductivity & $c_{p_{c}}$ & $1.13410^{3}$ & $\mathrm{~J} . \mathrm{kg}^{-1} \cdot \mathrm{K}^{-1}$ \\
Thermal diffusion coefficient in the tooling & $\beta_{t}$ & $0.3 \beta_{c}$ & $\mathrm{~m}^{2} . \mathrm{s}^{-1}$ \\
Mass ratio of resin in the composite & $m_{r}$ & 46 & $\%$ \\
Enthalpy of reaction & $\left(-\Delta H_{r}\right)$ & $8.52510^{4}$ & $\mathrm{~J} . \mathrm{kg}^{-1}$ \\
Pre-exponential factor & $A_{c}$ & $1.23310^{21}$ & $\mathrm{~s}^{-1}$ \\
Activation energy factor & $E_{a}$ & $1.67410^{5}$ & $\mathrm{~J} . \mathrm{mol}^{-1}$ \\
Perfect gas constant & $R$ & 8.314 & $\mathrm{~J} . \mathrm{mol}^{-1} . \mathrm{K}^{-1}$ \\
Exponential factor & $m$ & 0.524 & $(-)$ \\
Exponential factor & $n$ & 1.476 & $(-)$ \\
\hline
\end{tabular}

where:

$$
\begin{aligned}
& \beta_{t}=\frac{k_{t}}{\rho_{t} c_{p_{t}}} \\
& \beta_{c}=\frac{k_{c}}{\rho_{c} c_{p_{c}}}
\end{aligned}
$$


Geometrical Data are:

\begin{tabular}{l|c|c|c}
\hline Property & Name & Value & Unit \\
\hline \hline Tooling thickness & $z_{t}$ & $110^{-2}$ & $m$ \\
Half thickness of the polymer layer & $z_{c}$ & $1.2710^{-2}$ & $m$ \\
\hline
\end{tabular}

\section{References}

[1] Armaou, A., \& Christofides, P.D. (2002). Dynamic optimization of dissipative PDE systems using nonlinear order reduction. Chem. Eng. Sci., 57(24), 50835114.

[2] Balas, M.J. (1998). Stable feedback control of linear distributed parameter systems: time and frequency domain conditions. J. Math. Anal. Appl., 225(1), $144-167$.

[3] Bendersky, E., \& Christofides, P.D. (2000). Optimization of transport-reaction processess using nonlinear model reduction. SChem. Eng. Sci., 55, 4349-4366.

[4] Berglund, L.A. \& Kenny, J.M. (1991). Processing Science for High Performance Thermoset Composites. SAMPE Journal, 27(2),27-37.

[5] Bogetti, T.A. \& Gillepsie, J.W. (1991). Two-dimensional Cure Simulation of Thick Thermosetting Composites. J. Compos. Mater., 25, 239-233.

[6] Bogetti, T.A. \& Gillepsie, J.W. (1992). Process-induced Stress and Deformation in Thick-section Thermoset Composite Laminates. J. Compos. Mater., 26-5, $626-660$.

[7] Choi, J.H. \& Lee, D.G. (1995). Expert Cure System for the Carbon Fiber Epoxy Composite Materials. J. Compos. Mater., 29(9), 1181-1200. 
[8] Christofides, P.D., \& Daoutidis, P. (1997). Finite-dimensional control of parabolic PDE systems using approximate inertial manifolds. J. Math. Anal. Appl., 216(2), 398-420.

[9] Christofides, P.D. (1998). Robust control of parabolic PDE systems. Chem. Eng. Sci., 53(16), 2949-2965.

[10] Ciriscioli, P.R., Wang Q. \& Springer, G.S. (1992). Autoclave Curing Comparisons of Model and Test Results. J. Compos. Mater., 26(1), 90-102.

[11] Dufour, P., Couenne, F., \& Touré, Y. (2003). Model predictive control of a catalytic reverse flow reactor. special issue of IEEE Trans. on Control Syst. Technol. on Control of Industrial Spatially Distributed Parameter Processes, $11(5)$, in press.

[12] Dufour P., Touré Y., Blanc D., \& Laurent P. (2003). On nonlinear distributed parameter model predictive control strategy: online calculation time reduction and application to an experimental drying process. Comput. $\mathcal{G}$ Chem. Eng., in press.

[13] Eaton, J.W., \& Rawlings, J.B. (1990). Feedback control of chemical processes using online optimization techniques. Comput. \& Chem. Eng., 14(4-5), 469-479.

[14] Eder, H.H. (1999). MBPC Benefits and Key Success Factors. Proc. of the ECC, Paper F1033-6.

[15] El-Farra, N.H., Armaou, A., \& Christofides, P.D. (2003). Analysis and control of parabolic PDE systems with input constraints. Automatica, 39(4), 715-725.

[16] Fletcher, R. (1987). Practical Methods of Optimization. John Wiley and Sons.

[17] Godasi, S., Karakas, A., \& Palazoglu, A. (2002). Control of nonlinear distributed parameter processes using symmetry groups and invariance conditions. Comput. \& Chem. Eng., 26(7-8), 1023-1036. 
[18] Hanks, D.E., Lee, M.C., Young, R.C. \& Tajima, Y.A. (1988). Processing Science of Thick-section Composites. SAMPE Quarterly, 19(2), 19-28.

[19] Hoo, K.A., \& Zheng, D. (2001). Low-order control-relevant models for a class of distributed parameter systems. Chem. Eng. Sci., 56(23), 6683-6710.

[20] Irizarry-Rivera R., Seideer, W.D. (1997). Model-predictive control of the Czochralski crystallization process Part I. Conduction-dominated melt. J. of Crystal Growth, 178(4), 593-611.

[21] Joseph, B., Hanratty, F.W. \& Kardos, J.L. (1995). Model-Based Control of Voids and Product Thickness During Autoclave Curing of Carbon/Epoxy Composite Laminates. J. Compos. Mater., 29(8), 1000-1024.

[22] Kalra, L., Perry, M.J. \& Lee, L.J. (1992). Automation of Autoclave Cure of Graphite-Epoxy Composites. J. Compos. Mater., 26(17), 2567-2584.

[23] Kim, J.S. \& Lee, D.G. (1997). Development of an Autoclave Cure Cycle with Cooling and Reheating Steps for Thick Thermoset Composite Laminates. $J$. Compos. Mater., 31(22), 2264-2282.

[24] LeClair, S.R. \& Abrams, F.L. (1989). Qualitative Process Automation. Int. J. of Comp. Integrated Manufacturing, 2(4), 205-211.

[25] Loos, A.C \& Springer, G.S. (1983). Curing of Epoxy Matrix Composites. J. Compos. Mater., 17, 135-169.

[26] Loos, A.C \& Nagendra, S. (1989). Optimization of the Curing Process of Filament Wound Composites. Proc. of the 34th Int. SAMPE Symposium, 18531867.

[27] Luenberger, D.G. (1984). Introduction to Linear and Nonlinear Programming. Addison-Wesley. 
[28] Martinez, G.M. (1991). Fast Cures for Thick Laminated Organic Matrix Composites. Chem. Eng. Sci., 46(2), 439-450.

[29] McConnell, V.P. \& Strover, D. (1995). Advanced Composites: Performance Materials of Choice for Innovative Products. High-Performance Composites 1995 Sourcebook, 8-19.

[30] Michaud, D.J., Beris, A.N. \& Dhurjati, P.S. (2002). Thick-Sectioned RTM Composite Manufacturing, Part II. Robust Cure Cycle Optimization and Control. J. Compos. Mater., 36(10), 1201-1231.

[31] Palazolu, A., \& Karakas, A. (2000). Control of nonlinear distributed parameter systems using generalized invariants. Automatica, 36(5), 697-703.

[32] Patwardhan, A.A., Wright, G.T., \& Edgar, T.F. (1992). Nonlinear modelpredictive control of distributed-parameter systems. Chem. Eng. Sci., 47(4), 721-735.

[33] Pillai, V.K., Beris, A.N. \& Dhurjati, P.S. (1994). Implementation of ModelBased Optimal Temperature Profiles for Autoclave Curing of Composites Using a Knowledge-Based System. Ind. Eng. Chem. Res., 33, 2443-2452.

[34] Pillai, V.K., Beris, A.N. \& Dhurjati, P.S. (1996). Heuristics Guided Optimization of a Batch Autoclave Curing Process. Comput. Chem. Eng., 20(3), 275-294.

[35] Qin, S.J. \& Badgwell, T.A. (1996). An Overview of Industrial Model Predictive Control Technology. 5th International Conference on Chemical Process Control, $232-256$.

[36] Qin, J.S, \& Badgwell, T.A. (2003). A survey of industrial model predictive control technology. Control Eng. Pract., 11(7), 733-764.

[37] Rawlings, J.B. (2000). Tutorial Overview of Model Predictive Control. IEEE Control Systems, 20(3), 38-52. 
[38] Servais, R.A., Lee, C.W. \& Browning, C.E. (1986). Intelligent Processing of Composite Materials. SAMPE Journal, September/October 1986, 22, 14-48.

[39] Springer, G.S. (1988). The Role of Models in Manufacturing Thermosetting and Thermoplastic Matrix Composites. Proc. of Manufacturing International, Atlanta, GA, VI, 1-5.

[40] Thomas, M.M., Joseph, B. \& Kardos, J.L. (1997). Batch Chemical Process Quality Control Applied to Curing of Composite Materials. AIChE J., 43(10), $2535-2545$.

[41] Touré, Y., Biston, J., \& Gilles, G. (1994). Modeling of a distributed parameter process with a variable boundary: application to its control. Chem. Eng. Sci., $49(1), 61-73$.

[42] Touré, Y., \& Josserand, L. (1997). An extension of IMC to boundary control of distributed parameter systems. Proc. of the IEEE International Conference on Systems, Man and Cybernetics-CCS, Orlando, USA, 3, 2426-2431.

[43] Zheng, D., \& Hoo, K.A. (2002). Low-order model identification for implementable control solutions of distributed parameter systems. Comput. $\&$ Chem. Eng., 26(7-8), 1049-1076. 


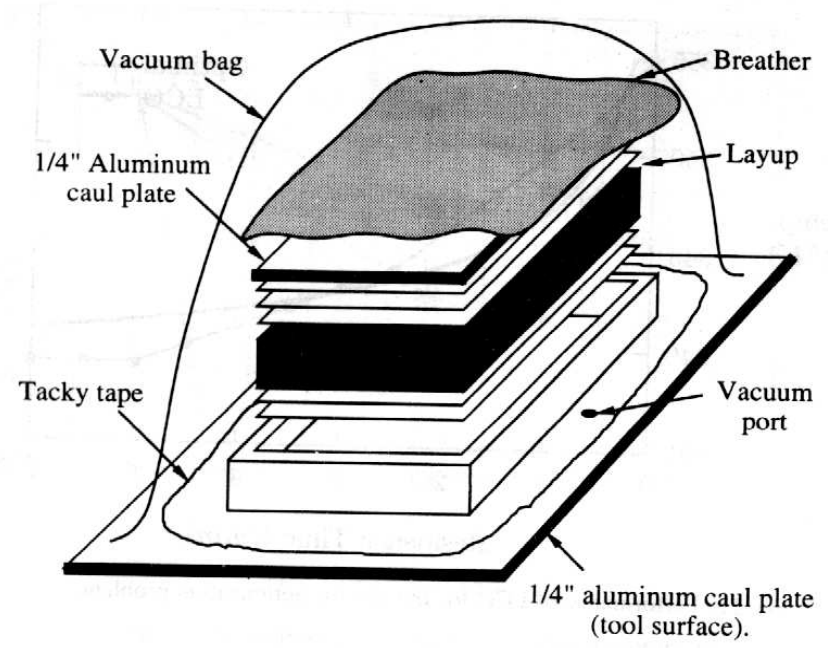

Fig. 1. Composite layup [34].

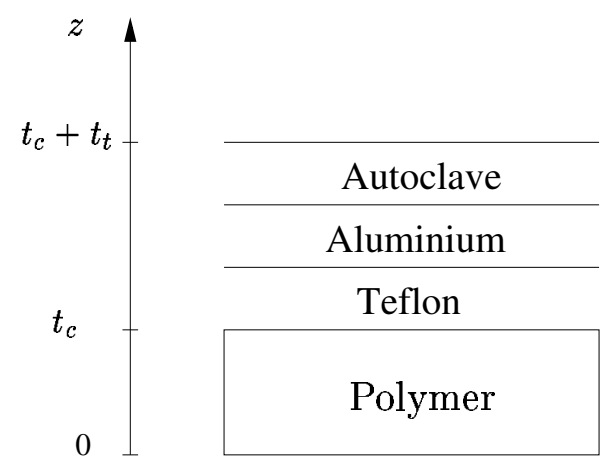

Fig. 2. Spatial domains from the center of the polymer to the edge of the autoclave.

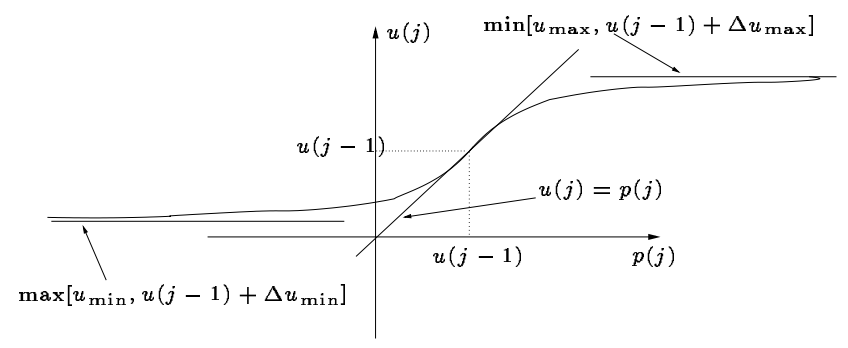

Fig. 3. Transformation law to handle manipulated variable constraints. 


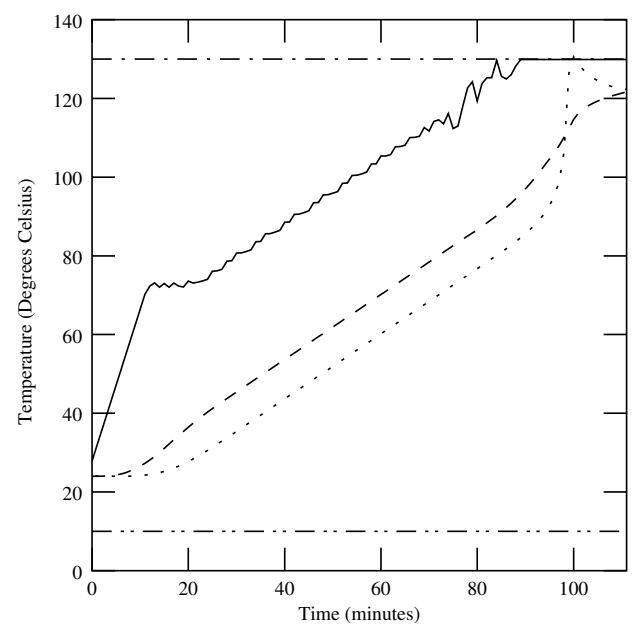

Fig. 4. Influence of the initial temperature difference upper constraint bound: temperatures in the autoclave (solid), at the surface (dashed) and at the center (dotted).

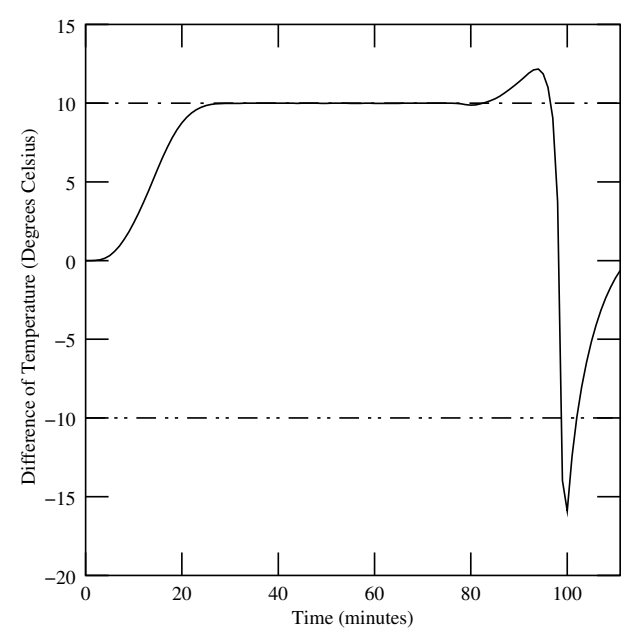

Fig. 5. Influence of the initial temperature difference upper constraint bound (dash-dot): temperature difference constraint between surface and center (solid). 


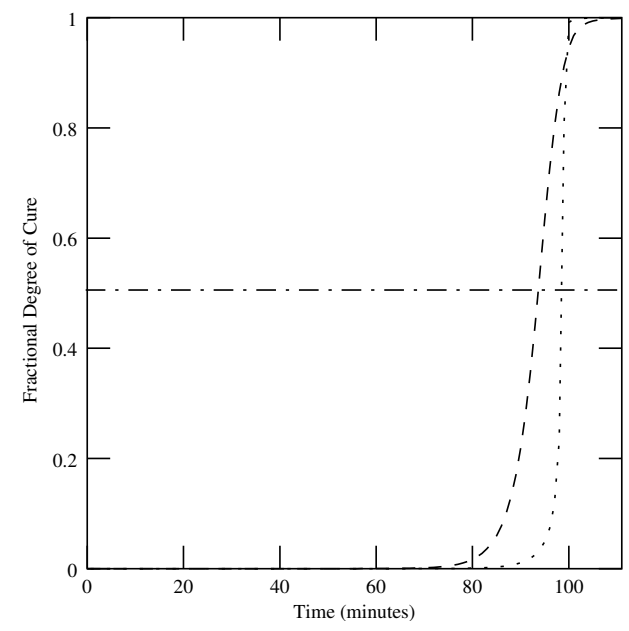

Fig. 6. Influence of the initial temperature difference upper constraint bound: fractional degrees of cure at the surface (dashed) and at the center (dotted).

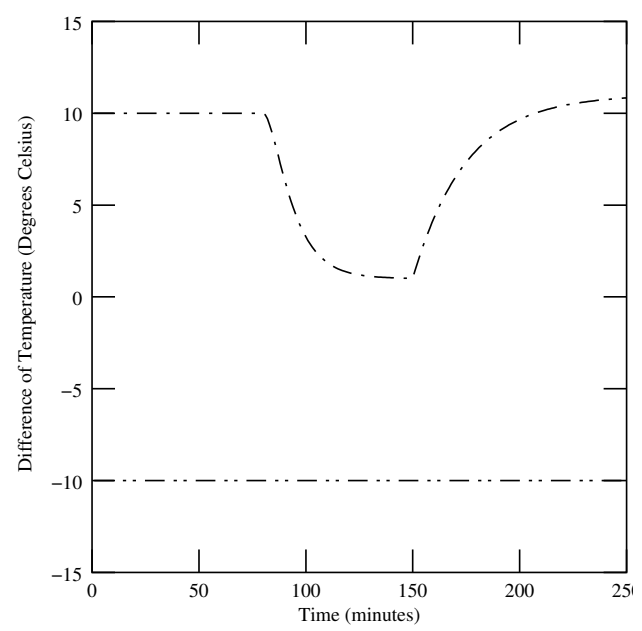

Fig. 7. Design of the bound for the temperature difference upper constraint (dash-dot) via "trial-feedback" strategy. 


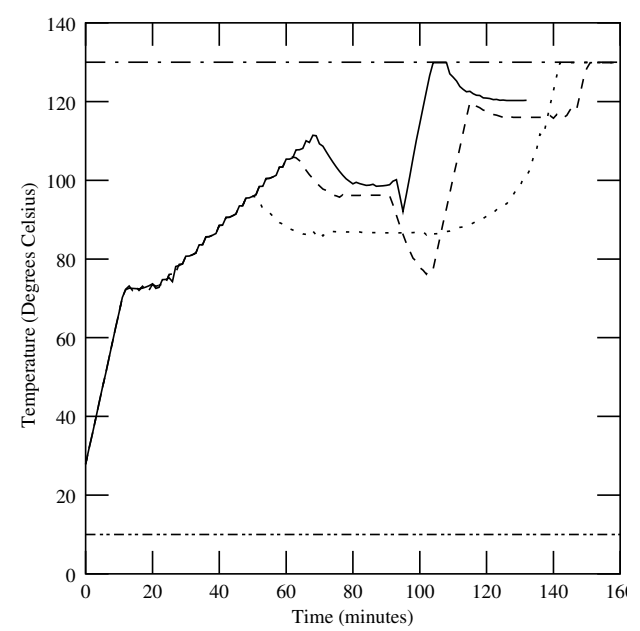

Fig. 8. Influence of the prediction horizon tuning over the temperature in the autoclave, in runs based on the designed temperature difference upper constraint bound: (solid: $\left.N_{p}=15\right),\left(\right.$ dashed: $\left.N_{p}=25\right),\left(\right.$ dotted: $\left.N_{p}=45\right)$.

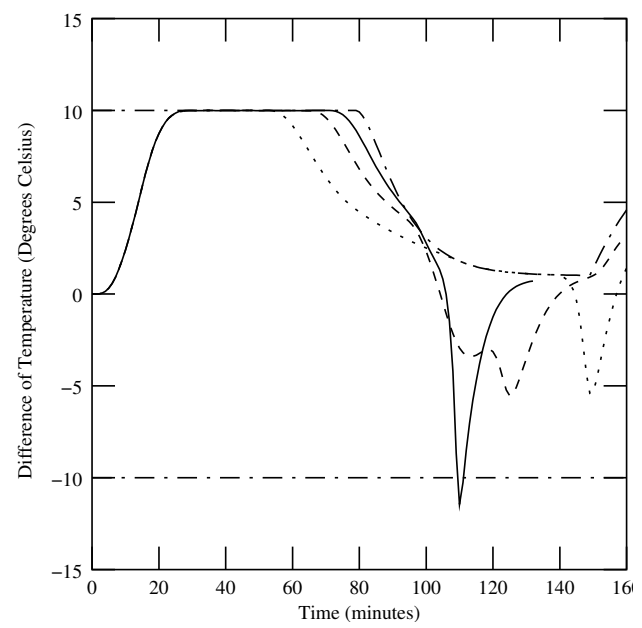

Fig. 9. Influence of the prediction horizon tuning over the temperature difference constraint between surface and center, in runs based on the designed temperature difference upper constraint bound: (solid: $\left.N_{p}=15\right)$, (dashed: $\left.N_{p}=25\right)$, (dotted: $\left.N_{p}=45\right)$. 


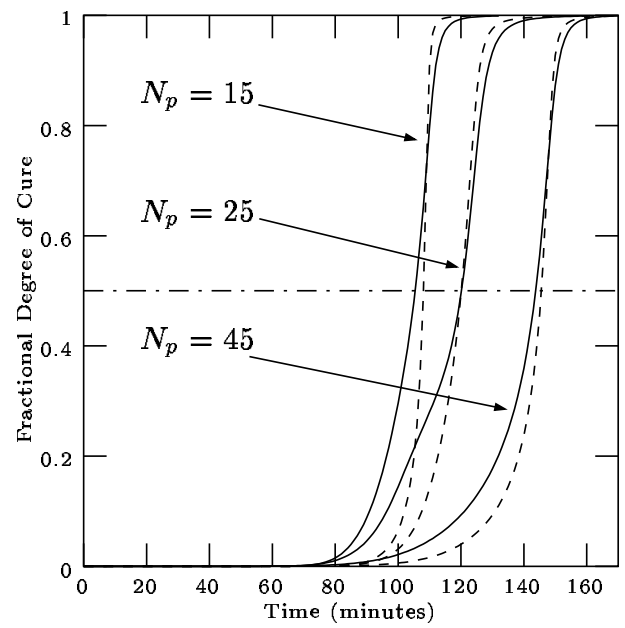

Fig. 10. Influence of the prediction horizon tuning over the fractional degrees of cure at the surface (solid) and at the center (dashed), in runs based on the designed temperature difference upper constraint bound. 